\title{
Quicktone - Ein Interview mit Ivan Thoralf Rosenqvist
}

Eingegangen: 28 Mai 2004 / Angenommen: 1 Juni 2004 / Online veröffentlicht: 12 August 2004 (C) Springer-Verlag 2004

\begin{abstract}
Abstrakt Durch Vermittlung von Jörn Thiede gelangte das Geologenarchiv in den Besitz eines Videobands von einem Fernsehinterview mit Rosenqvist (auch gelegentlich Rosenquist), das Elen Roaldset (E.R.) im Geologischen Institut der Universität Oslo am 8.September 1988 führen konnte. Es umfasst den gesamten Lebenslauf und die wichtigsten wissenschaflichen Leistungen dieses aussergewöhnlichen Geologen und Mineralogen. Er wurde in Wien am 17. Mai 1916 geboren, kam aber mit seinen Eltern schon 1917 nach Oslo, wo er am 8. Oktober 1994 starb (K. Bjørlykke 1995).

Im Folgenden sollen aus dem Interview nur seine wegweisenden Untersuchungen der Quicktone, seine fachliche Vielseitigkeit, sein gesellschaftliches Engagement und seine schwierigen Erfahrungen mit Deutschland herausgegriffen werden. Das in der Landessprache geführte Gespräch wurde von Geir Ebelfoff und Lars Erik Kastilan ins Deutsche transkribiert.
\end{abstract}

\section{Die Quicktone}

Rosenqvist sagte dazu: „Mit den Untersuchungen an den Quicktonen hatte ich Glück, glaube ich. Ich hatte vor allen anderen Geowissenschaftlern den Vorteil, dass ich mich in der Chemie besser auskannte als sie. Das Phänomen der Quickone ist, dass man von einem festen Zustand ausgeht, der bei einer mechanischen Belastung in einen flüssigen übergeht. Dann bleibt er weiterhin flüssig, bis er austrocknet, Wasser verliert. Dieses Phänomen war beschrieben, aber überhaupt nicht verstanden worden. Und mich, der Oberflächenchemie und Kolloidphysik bis $\mathrm{zu}$ einem gewissen Grad beherrschte, hatte das sehr interessiert. Ich kam in Kontakt mit dem sich am schnellsten verändernden Ton, der bis jetzt beschrieben worden ist, bei Asrumvannet i Hedrum. Und dort fand ich eine Erklärung seines Verhaltens. Das hängt damit zu-

E. Seibold $(\bullet) \cdot$ I. Seibold

Richard-Wagner-Str. 56, 79104 Freiburg, Deutschland

E-Mail: Seibold-Freiburg@t-online.de sammen, dass er ursprünglich eine marine Ablagerung war. Als dann das Land aus dem Meer stieg, hatte sich Regenwasser aus den benachbarten Bergen durch den Ton gedrängt und hatte die Salze herausgewaschen. Wir haben nämlich herausgefunden, dass der Ton sehr salzarm war und wir wussten ja, dass das Porenwasser ursprünglich den gleichen Salzgehalt wie das Meerwasser gehabt haben musste. Ich konnte die Zusammenhänge erklären. Wenn man die Salze und damit auch die Kationen entfernt - und diese kompensieren die negativen Ladungen des Tons, - so wird alles negativ geladen. Damit beginnen die abstossenden Kräfte so groß zu werden, dass die Bindungen gebrochen werden. Die Minerale wollten sich nicht mehr zusammenbinden wie in normalen Tonen. Alle Tone werden zwar ein wenig weicher, wenn man sie mechanisch beansprucht, aber diese werden flüssig." (Interview). „Kvikk“ leirer sind also "muntere, lebhafte“ Substanzen, so wie auch das „Queck"silber.

Marine Tone, die nach Entlastung durch das $\mathrm{Ab}$ schmelzen des Eises, also postglazial herausgehoben werden, gibt es nicht nur in Norwegen, Schweden und Nordrussland, sondern auch in Ostkanada, Alaska und stellenweise auch in Neuseeland. In Norwegen hat man sich schon besonders früh um ihr Verständnis bemüht. [V. M. Goldschmidt 1926, Rosenqvist vor allem 1946a und b, 1953, 1955, (deutsch 1956), 1958, 1959, 1966 und 1977, L. Bjerrum 1954]. -Neuere Zusammenfasssungen finden sich bei J. K. Torrance 1983 und, Methodisches zur Bestimmung von Mikrogefügen in Tonen, bei R. H. Bennett et al (1991).

Um den Ansatz und die Erfolge Rosenqvists in diesem Bereich besser verstehen zu können, sei im Folgenden auf einige wissenschafliche Voraussetzungen näher eingegangen.

Die Basis von Rosenqvists obiger Erklärung beruht also darauf, dass sich die Tone, die generell aus Partikeln unter $2 \mu$ zusammengesetzt sind, wie kolloidale Systeme verhalten, in denen hohe Oberflächenenergien bestimmend sind. Schon am Anfang des 19. Jahrhunderts war zudem in Versuchen gezeigt worden, daß Tontrübe zur 
positiven Elektrode wandert, also negativ geladen ist. Beides ist auch bei den Quicktonen wichtig.

\section{Material}

In Norwegen sind diese keine reinen Tone. Sie enthalten stets Silt. Mineralogisch sind daran beteiligt Quarze, Feldspäte, dunkle Minerale und dazu Tonminerale, vor allem Illit und Chlorit- Das sind alles Abtragungsprodukte des dortigen Grundgebirges. Auch in Kanada gehören nur rund $60 \%$ der dortigen Quicktone der Tonfraktion an, die neben weniger als 30\% Tonmineralen die obigen Minerale enthalten (J. K. Torrance 1991, 550).

\section{Sensitivität}

Die Quicktone haben eine hohe Sensitivität, reagieren daher auf Beanspruchungen sehr empfindlich. Die Sensitivität kann im Labor durch Scherversuche bestimmmt werden als Quotient von Scherfestigkeit der ungestörten Probe und der gestörten, d.h. durchgekneteten (remolded) Probe. Für Quicktone werden Werte um und grösser als 30 angegeben. (Rosenqvist 1953, Norsk Geoteknisk Forening 1974). Die A r t der Tonminerale scheint auf das Verhalten weniger Einfluss zu haben als die Korngrössenverteilung. Wichtig ist der Grenzbereich Ton/ Silt (L. L .Smalley 1971, 310, J. K. Torrance 1983, 550).

Noch wesentlicher ist das M i k r o gefüge der Tone. Die klassischen Untersuchungen von K. Terzaghi (1925), V. M. Goldschmidt (1926) und A. Casagrande (1932) haben gezeigt, dass in ihnen „Bienenwaben“- oder „Kartenhaus“-Gefüge vorliegen. Minerale kleben dabei an Kontakten durch Adhäsion zusammen und lassen dazwischen grosse Hohlräume. Dies konnte Rosenqvist (1955) mit etwas undeutlichen Bildern mit optischen Mikroskopen wahrscheinlich machen. Einen Quantensprung bei diesen Untersuchungen brachte aber der Einsatz des Transmissions-Elektronenmikroskops (TEM). Nach sorgfältiger Präparation konnte durch Stereophotographie nicht nur die Kartenhausstruktur illustriert, sondern auch verschiedene Kontaktmöglichkeiten gezeigt werden, etwa Ecke/Ecke, Ecke/Fläche, Kante/Fläche oder Fläche/Fläche (Rosenqvist 1958 und 1959, s. Abbildung 1). Schliesslich ergab sich, dass die Partikel vor allem in Stapeln (domains) zusammengeschlosssen sind. Weitere Methoden, etwa die Verwendung des Raster-Elektronenmikroskops (B. Mattiat 1969, R. E. Ferrell 1987 oder Ferrell \& P. K. Carpenter 1991, s. Abbildung 2) führten zu verfeinerten Bildern wie im Atlas von N. R. O'Brien \& R. M. Slatt (1990).

\section{Flockung}

Sedimentationsversuche ergaben, dass die Kartenhausstruktur dadurch entsteht, dass das suspendierte Material im Meerwasser als Flocken zu Boden sinkt (s. Abbildung

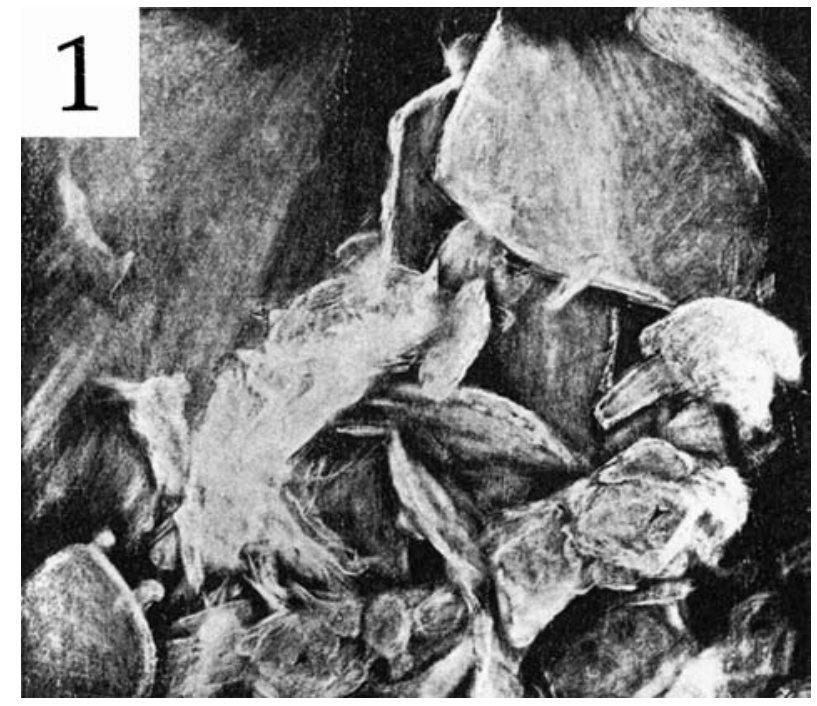

Abbildung 1: Anordnung der Minerale im Quickton (Blauer OsloTon), Unterrand rund $4 \mu$. Aus I.T.Rosenqvist 1958, 442, Abb. 5

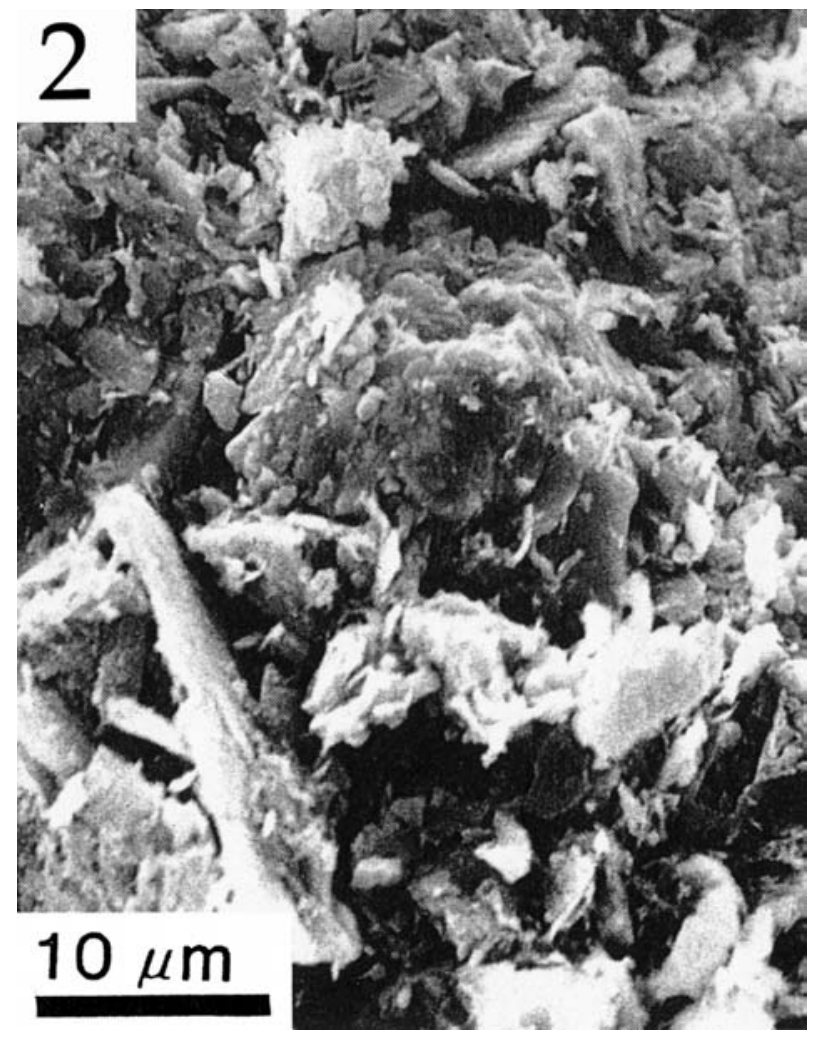

Abbildung 2: Quickton aus dem Oslofjord mit Illit und Chlorit und relativ offenem Mikrogefüge. Die Tonmineale zeigen eine typische Zufallsanordnung mit vielen Ecke/Fläche- Kontakten. Die großen-schwarzen-Poren erhöhen die Permeabilität. Aus R.E.Jr. Ferrell \& P.K.Carpenter 1991, 299, Abb. 32.2.D

3). Sie können bis Sandkorngrösse erreichen (K. Kranck, 1991). In den Flocken werden die Partikel vor allem durch elektrostatische und elektrochemische Kräfte zusammengehalten. Die im allgemeinen negativ geladenen, 

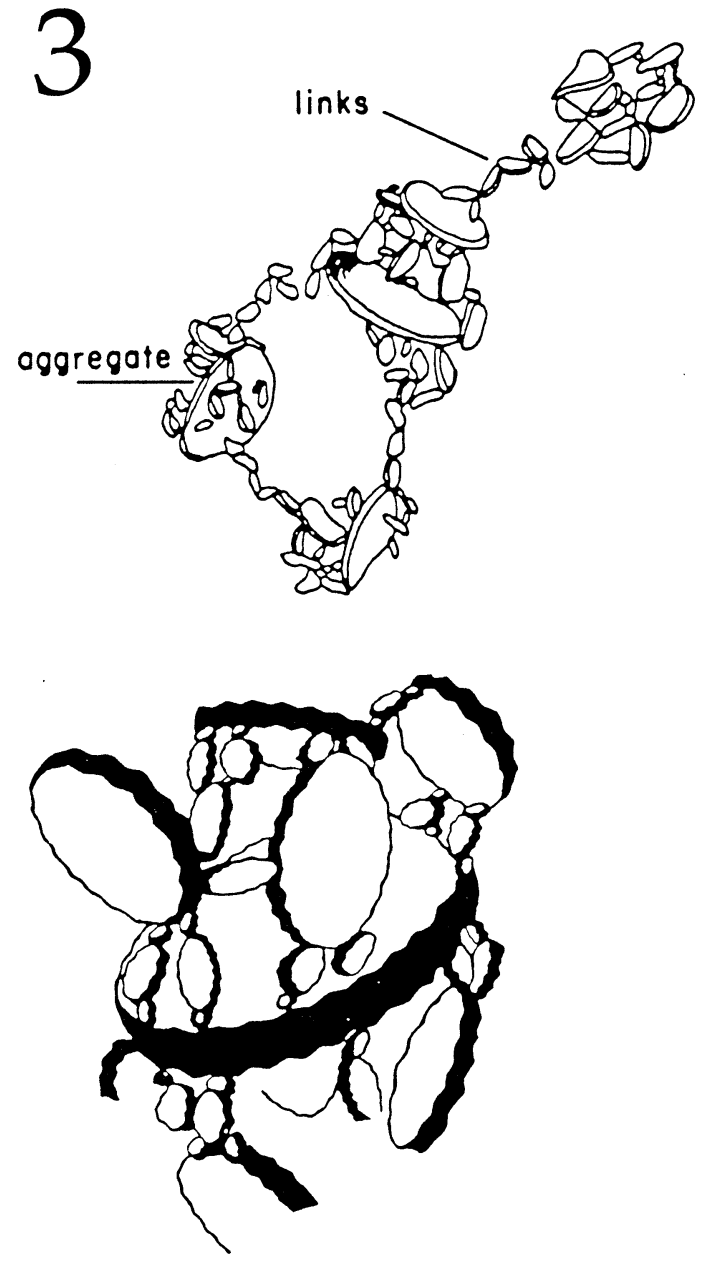

Abbildung 3: Beispiele für die Anordnung von Einzelmineralen (in $\mu$ - Dimensionen) in Flocken. Aus K. Kranck in R.H. Bennet et al 1991, 127, Abb. 12.2

also sich abstossenden Tonminerale und feinen Siltpartikel können sich durch die positiven Kationen von $\mathrm{K}^{+}$, $\mathrm{Na}^{+}, \mathrm{Ca}^{++}$und $\mathrm{Mg}^{++}$im Meerwasser neutralisieren und dadurch annähern. So nimmt beispielsweise nach Versuchen von W. von Engelhardt \& K. H. Gaida (1963) bei sämtlichen Tonmineralen die Kompaktionsrate direkt mit der NaCl-Konzentration zu. Die von der spezifischen Oberfläche abhängige Kationen-Austauschkapazität nimmt dabei vom Kaolinit über den Illit zum Monmorrillonit auf das bis zu Zehnfache zu (J. K. Mitchell, 1976).

In marinen Flachwassertonen ist allgemein organische Substanz $\left(\mathrm{C}_{\text {org }}\right)$ angereichert, da sich diese hydraulisch ähnlich verhält wie Tone. Deshalb ist mit deren Einfluss auch bei der Flockung zu rechnen, auch unter Beteiligung von Bakterien. Kotpillen mit ihren für die Tiefseesedimente so wichtigen erhöhten Sinkgeschwindigkeiten spielen gleichfalls eine Rolle.

Diese Hydratationsprozesse spielen sich an den Kontakten im Angstrøm- Bereich ab, also in molekularen Dimensionen (W. Drost-Hansen 1991). Man nimmt dabei eine Doppellage an, wobei ein negativ geladener
Wasserfilm das Mineral umhüllt (H. H. Rieke \& G. V. Chilingar 1974, 229). Rosenqvist (1958, 450) ordnet diesen Film sogar direkt der Mineralphase zu, was er mit Austauschversuchen unter Verwendung von Isotopen stützt. Die zweite Lage darüber ist durch Kationen positiv geladen. Wenn diese Doppellage durch höhere Kationenkonzentration zusammengedrückt wird, sollen die van der Waals-Kräfte stärker werden als die sich abstossenden Coulombschen Kräfte.

\section{Salzwasser versus Süsswasser}

Es ist noch eine offene Frage, ob auch im Süsswasser nennenswerte Flockung als Voraussetzung für die Bildung von Kartenhausgefügen und damit von Quicktonen auftritt. Eventuell spielen dort die zweiwertigen Kationen die wichtigste Rolle, oder auch organische Substanzen (J. K. Torrance bei schwedischen Süsswasser-Quicktonen 1983, 552).

Das Gefüge von Süsswassertonen scheint generell noch weiterer Forschung zu bedürfen. Auf der einen Seite wird angenommen, dass die Partikel darin streng parallel, auf alle Fälle besser ausgerichtet sind als in marinen Tonen, da sie aus dispersen Suspensionen stammen sollen (Rosenqvist 1959, C. F. Moon 1972). Von anderen wird für sie eine „zufällige“ Anordnung angegeben, die zu massiven, blockigen Tonsteinen (Claystones. Mudstones) führen soll. Generell könne man Tonsteine mit ihrem ungeregelten Mikrogefüge von Schiefern (shales) mit ihrer überwiegenden oder doch deutlich besseren Orientierung der plättchenförmigen Partikel unterscheiden (N. R. O“Brien \& R. M. Slatt 1990). Dieser Unterschied ist in Karbonprofilen schon früh aufgefallen (I. E. Odom 1967, D. A. Spears 1976). Das blockige Gefüge der zwischen den spaltbaren marinen Schiefern eingebetteten, oft meterdicken „Underclays“ wurde aber durch Bodenbildung und Homogenisierung durch Wurzeln geprägt.

Die umgekehrte Frage, ob marine Tone durch intensive Bioturbation ihr ursprüngliches Kartenhausgefüge verlieren können (M. Gipson 1966) und damit zu Tonsteinen werden, ist noch offen. Die terrestrischen, oft viele Meter mächtigen Abfolgen unserer deutschen Keupermergel und -tone geben gleichfalls noch Fragen auf. Es verwundert deshalb nicht, dass 1991 eine der dringlichsten Forderungen der in einem Workshop versammelten Spezialisten für Tongefüge war, aus ihren Befunden das frühere Ablagerungsmilieu besser abzuleiten (M. H. Hulbert 1991, 565).

In diesem Zusammenhang bleiben die Flussmündungen ins Meer von besonderem Interesse (J. P. M. Syvitski 1991). Dies vor allem bei solchen mit Gezeiteneinfluss. Die bei der Durchmischung des Fluss- mit dem Meerwasser ausgeflockten Suspensionen wandern bekanntlich in der Girondemündung als ,bouchon vaseux“ hin und her und verschlammen Häfen und Schleusen. Am Boden des Hafens von Rotterdam findet sich beispielsweise ein solcher flüssiger Schlamm, der zu $80 \%$ aus Flocken besteht (K. Kranck 1991,107). 
Verflüssigung

Werden diese marinen Tone aus dem Meer herausgehoben, können die stabilisierenden Kationen durch Regenwässer ausgewaschen werden. Das Kartenhausgefüge bleibt dabei intakt, wird aber äusserst instabil (Rosenqvist 1953, C. F. Moon 1972, 211). Auf Faktoren wie die Rolle organischer Substanzen, die Auflast durch weitere Sedimentation und nach der Heraushebung die Verwitterung mit Pyritbildung und den Folgen nach deren Oxydation, Trockenrisse, Durchwurzelung und ähnliches kann hier nicht eingegangen werden.

Werden diese ausgelaugten Tone durch mechanischen Einfluss, etwa durch Erschütterungen durch Maschinen oder Verkehr oder Erdbeben gestört, so bricht das Gefüge zusammen. Die Tone werden spontan flüssig. Die Partikel verlieren ihren Kontakt und sinken im Porenwasser als Suspension $\mathrm{ab}$.

\section{Verfestigung von Quicktonen}

Was tun bei einem solchen Ereignis? Hier soll wieder Rosenquist zu Wort kommen (Interview):

„Im Jahre 1952 bekam ich die Stelle des Forschungsleiters am Norwegischen Geotechnischen Institut (NGI). Dadurch, dass es ein rein praxisorientiertes Forschungsinstitut war, hatte ich eine relativ gute Ausrüstung zur Verfügung, viel besser als sie die Universität jemals hatte. So wurde es dort eine sehr amüsante Arbeit und ich konnte unter anderem auch an den Quicktonen weiterarbeiten...

\section{E.R.: Es verging ja nicht so lange Zeit,} bevor du deine Theorie in die Praxis umsetzen konntest!

Ja, der erste Erdrutsch ereignete sich 1953 auf dem Mosseveien $<$ Strasse in Oslo $>$. Die meisten erinnern sich wohl nicht mehr daran. Der Mosseveien draussen auf dem Baekkelaget rutschte weg und einige Menschen kamen dabei ums Leben. Und da gab es dann dort,wo die Strassse vorher war, einen offenen Graben mit flüssigem Quickton.

\section{E.R.: Ging nicht auch ein Teil der Bahngeleise verloren?}

Ja, ein kleiner Teil. Aber der ganze Verkehr auf dem Mossevei wurde blockiert und musste über den EkebergHügel umgeleitet werden. So wurde ich von Bjerrum $<$ dem damaligen Direktor des Instituts $>$ gefragt ob ich dorthin fahren könnte, um mir das Ganze anzusehen und ob ich einen Plan hätte, wie es repariert werden könnte. Daraufhin kam ich um sieben Uhr morgens an die Rutschung. Ich erbat einen Bagger, Kies und Sand. Dann fuhr ich nach Oslo hinein und kaufte, als die Geschäfte öffneten, sechs Säcke Salz, 600 Kilogramm. Ich habe sodann das Salz mit dem Bagger in die Tonsuppe einmischen lassen. Sie ist anfangs nur die Bagerschaufel heruntergeflossen, doch das Graben wurde zunehmend schwerer und schwerer. Anfänglich war alles so flüssig, dass man keinen Stein in der Grösse eines Zuckerwürfels drauflegen konnte, ohne dass dieser einsank. Nach einiger Zeit konnten wir über das fester werdende Material Sand und schliesslich Kies schichten. Der Verkehr konnte wieder aufgenommen werden. Zum Mittagessen kam ich in das Institut und dort fragte mich Direktor Bjerrum „Hast du schon Pläne für eine Lösung?“ „Vor einer halben Stunde ist schon ein sechs Tonnen schwerer Lastwagen darübergefahren" sagte ich. Das hat viel Aufmerksamkeit erregt, denn es gab Journalisten, die das beobachtet hatten, da es ja kurz nach dem Erdrutsch passiert war. So wurde ich mehrmals von Zeitungen interviewt. Es war ja auch wirklich frappierend $\mathrm{zu}$ sehen, wie eine flüssige Tontrübe im Lauf von eineinhalb Stunden nach der Einmischung des Salzes stabilisiert werden konnte. Das Strassenbauamt hatte Mitarbeiter hochgeschickt, als ich in Oslo war und Salz kaufte. Es hat aber lediglich beobachtet.

\section{E.R.: Doch, das hatte auf viele einen grossen Eindruck} gemacht. Besonders interessant war ein Leitartikel des darauf folgenden Tages in der Zeitung „Vårt Land“.

Ja, ich wurde von der Zeitung „Verdens Gang“ oder so ähnlich interviewt und erklärte die Wichtigkeit der Kationen, der positiven Ionen, die die abstossenden Kräfte neutralisieren. Und so fand es diese christliche Tageszeitung „Vårt Land“ interessant und hat darüber berichtet. Unter anderem hiess es darin, „dass vor 2000 Jahren der, von dem wir unsere Zeitrechnung haben, auf einem -Berg in Galilea stand. Er sagte zu seinen Jüngern „Ihr sollt das Salz der Erde sein“. Diese Worte waren bis vor kurzem noch unverständlich bis Dr. Rosenqvist vom Geotechnischen Institut mit einer Erklärung kam“. Meine gesamten Ausführungen haben sie dann heruntergeschrieben. Sie sagten, unsere abendländische Kultur sei ein imponierendes Bauwerk, aber wenn die negativen Kräfte die Überhand gewinnen, kann alles zusammenfallen. „Ihr, die ihr das Wort der Erlösung habt-und wir leben ja in einem christlichen Land-ihr habt das Salz der Erde zu sein, kommt und stabilisiert! Es muss ja gar nicht viel sein. Auf dem Mossevei hat man nur sechs Säcke gebraucht." Das war das erste und einzige Mal, dass ich als lebender Beweis für diese Sichtweise in einem Leitartikel erschien. Nur weiss ich selbst nichts damit anzufangen."

\section{Zur Person}

Als erstes sei auf die fachliche Vielseitigkeit Rosenqvists hingewiesen. Schon als Student an der Universität von Oslo wurde Rosenquist durch den Mineralogen und Begründer der modernen Geochemie, Victor Moritz Goldschmidt (1888-1947) besonders gefördert. „Er war der größte Naturforscher Norwegens" (Interview). In der 
Chemie war es dort vor allem eine Schülerin von Madame Curie, Ellen Gledlitsch, die einen Aufenthalt in Paris im Jahre 1937 anregte. In Paris lernte er mit der Radioaktivität umzugehen, wonach er Isotope als Tracer bei geologischen Prozessen verwenden konnte. (E. Føyn \& Rosenqvist 1938). 1940 erwarb er den Magistergrad.

Seine gründliche chemische und physikalische Ausbildung trug in der Folge reiche Früchte. 1941 kam er am norwegischen Straßenbauamt in Kontakt mit der Ingenieurgeologie und schon 1942 publizierte er eine Arbeit über Tone in Norwegen. Daraus erwuchs seine Erforschung der Quicktone. Wie dort schon ging er auch danach die Probleme vom Prinzipiellen her und möglichst quantitativ an, etwa bei der Metamorphose oder der Verwitterung und später beim „Nummedal-Projekt“, einer Studie der Erosions- und Sedimentationsprozesse in einem norwegischen Flußsystem. Sein Interesse am heimischen Gelände geht auch aus seiner Beteiligung an der Uranprospektion hervor.

Dies alles vollzog sich nach dem Krieg in den unterschiedlichsten Positionen und Institutionen, etwa als Leiter der Chemischen Abteilung im Militärforschungsinstitut, als wissenschaftlicher Mitarbeiter am Geologischen Institut der Universität Bergen, als Forschungsleiter im Norwegischen Geotechnischen Institut in Oslo und seit 1956 als Teilzeit-Professor (Professor II) für Sedimentologie und Geotechnik an der dortigen Universität. $\mathrm{Ab}$ 1965 war er bis zu seiner Emeritierung 1983 Lehrstuhlinhaber für Mineralogie am Geologischen Institut der Universität Oslo. Hier konnte er in engstem Kontakt Studenten im Gelände wie auch im Labor für seine Auffassungen begeistern.

International wurde er, wie erwähnt, durch seine Erfolge in der Anwendung physikalischer und chemischer Methoden auf das Verhalten von Tonen besonders bekannt. So wurde er 1960-1966 Präsident, dann Ehrenmitglied der Association internationale pour l'étude des argiles (AIPEA) im Rahmen der International Union of Geological Sciences (IUGS). Zum Höhepunkt für ihn wurde 1977 die einschlägige Konferenz in Oslo, bei der er das Grundsatzreferat hielt.

Sein berufliches Hin und Her zwischen Theorie und Praxis mit den unterschiedlichsten Fragestellungen spiegelt sich in seinem Schriftenverzeichnis von 250 Titeln wider. In 42 davon zeichnete er als Co-Autor. über 50 davon hatten das Verhalten der Tone zum Gegenstand (K. Bjørlykke 1995).

Stets betonte er dabei die Bedeutung der Grundlagenforschung als Basis für jede Anwendung. Nur wer die beiteiligten Prozesse kenne, könne Fälle in der Praxis beurteilen und Vorhersagen wagen

Seine fachlichen Kenntnisse setzte er auch unerschrocken bei gesellschaftlichen Fragen ein.

„Dies hat mich in große Schwierigkeiten gebracht. Aber ich finde es wichtig, dass Wissenschaftler die Wahrheit sagen und davon reden, wovon sie eine Ahnung haben. Auch dass man seine Kenntnisse der Gesellschaft zur Verfügung stellen sollte, freiwillig, und nicht wegen des Geldes. Man sollte auch nicht etwas sagen, weil es ein Politiker hören will“" (Interview).

Er nahm, oft kämpferisch gegen den Zeitgeist, zu verschiedensten Problemen Stellung. Häufig genug wurde er deshalb scharf angegriffen. So beleuchtete er die Rohstoffsituation im allgemeinen, wobei er vor allem den Begriff „Reserven“ klar definierte, und im besonderen bei den Kohlenwasserstoffen unter der Nordsee. Er äusserte sich zur Welternährung und $\mathrm{zu}$ verschiedensten Umweltfragen und war Mitglied bei Pugwash. Ausführlich und recht kontrovers ging er beispielsweise auf den Sauren Regen ein. Nicht selten zog er auch klare politische Folgerungen. So schrieb er im März 1971 an den damaligen Vorsitzenden der Arbeiterpartei, Trygve Brattelli (1910-1984): „Jetzt, da Du Ministerpräsident geworden bist, gibt es eine Sache, die ich jetzt für sehr wichtig halte und in der ihr etws tun solltet. Gut verdienende Industriebetriebe sollten nicht die Erlaubnis bekommen, den Gewinn an die Eigentümer zu verteilen, wenn sie umweltschädliche Abfälle verursachen... Es war meine Meinung, dass man hinter sich aufräumen müsse“ (Interview).

In gleich direkter Weise stellte er sich gegen die Mitgliedschaft seines Landes bei der NATO oder bei der Europäischen Gemeinschaft, die ja damals von Brattelli befürwortet wurde.

Dieses politische Engagement zeigte sich schon früh. Er machte sich schon 1937 in Paris Vorwürfe, dass er sich nicht als Freiwilliger am spanischen Bürgerkrieg beteiligte, sondern nach Oslo zurückging (Interview). Dafür setzte er sich 1939 als Ingenieuroffizier und Skilehrer in Finnland im Krieg gegen die Sowjetunion ein.

Als die Wehrmacht am 9. April 1940 Norwegen besetzte, nahm er sofort aktiv an den Kämpfen im Osten des Landes teil und ging dann in den Untergrund, wo er zuletzt Kurierchef für die Londonroute über Stockholm wurde. Das zivile Alibi war seine Tätigkeit im Straßenbaulabor. Am 8.Mai 1942 wurde er aber verhaftet und wegen „illegaler Aktivitäten, Spionage etc“ angeklagt. Sein Prozess sollte vor dem SS- und Polizeigericht Nord stattfinden (Norsk Krigslexsikon).

Er kam in das Konzentrationslager in Grini bei Oslo. „Ich saß 14 Monate in einer Einzelzelle mit weißgestrichenen Fenstern, so dass kein Norweger mich sehen konnte. Die Wärter, die Essen und so etwas brachten, setzten das Essen auf einen kleinen Schemel vor die Tür. Dann öffnete der Wärter die Tür, so dass ich das Essen hineinnehmen konnte... Ich hatte dort auch eine Toilette, wurde aber nie zum Lüften hinausgebracht. Ich saß dort und musss sagen, dass man Freude durch seine Ausbildung erfuhr. Andere Leute gingen kaputt, wenn sie allein sitzen mussten. Statt dessen hatten wir mit unserem Studium und Wissen mentale Bauklötze bekommen, mit denen wir auf ganz eigene Art spielen konnten.... Dank einer finnischen Empfehlung wurde ich relativ höflich behandelt. Ich durfte in der Zelle sitzen und bekam einiges an Literatur. Meine Frau schickte alle meine wissenschaftlichen Aufzeichnungen hoch. Dort konnte ich in Ruhe und Frieden sitzen, ohne von Telefon oder ähnli- 
chem gestört zu werden. Ich konnte meine Doktorarbeit zusammenfassen und verschicken... Es waren etwa 100 Seiten“ .(Interview).

Hilfreich waren in dieser Situation der damalige Rektor, Adolf Hoel und der Geologe an der Universität Oslo, Olaf Holtedahl(1885-1975). Im Juli 1943 wurde Rosenqvist indessen bis Kriegsende in das Konzentrationslager Sachsenhausen überstellt, wo damals rund 2500 andere Norweger eingesperrt waren. Es heisst dazu im Interview recht lakonisch: „Dort hatte ich eine ganz normale Zeit, aber als ich mit Graf Bernadotte nach Schweden kam, ging ich wieder direkt in den Militärdienst rein." Es ist erstaunlich, wie gelassen er auf diese fünf Jahre seines Lebens zurückblickte.

Dies kann auch durch eine stundenlange Unterhaltung mit E.S. in einem Schlafwagen am 7. Dezember 1964 bezeugt werden. Er brachte Exkursiosteilnehmer im Rahmen des 22. Internationalen Geologenkongress ' von Delhi in den Himalaya. In dieser Nacht war ich von dieser starken, unverwechselbaren Persönlichkeit tief beeindruckt. Er war ein überzeugter, idealistischer Sozialist, der auch über die vielen negativen Seiten der Systeme in der damaligen Sowjetunion und der Deutschen Demokratischen Republik unbeeinflussbar hinwegsah.

Im Nachruf (K. Bjørlykke 1995) heisst es: „In fachlichen und fachlich-politischen Diskussionen konnte Rosenqvist scharf sein, kompromisslos und polemisch auf eine Art und Weise, die auch provozieren konnte... Er versuchte allerdings niemals jemanden zu verletzen... Er zeigte oft ein starkes persönliches Engagement für seine Studenten, Kollegen und Freunde“.

Und der Schluss: „Ivan Th. Rosenqvist war ein Kollege, der in weiten Kreisen hoch anerkannt und geschätzt wurde. Wir haben keinen anderen, den man mit ihm vergleichen könnte und wir werden ihn sehr vermissen. Wir haben eines unserer in vielen Jahren bedeutendsten und markantesten Mitglieder < der Akademie> verloren. Wir werden ihn ehrend in Erinnerung behalten." (K. Bjørlykke 1995)

Danksagung Für die Möglichkeit, das Interview transkribieren zu lassen, bedanken wir uns bei Prof. Dr. Heinrich Anz und der Wissenschaftlichen Gesellschaft in Freiburg im Breisgau. Wiederum wurden uns für das Geologenarchiv in dankenswerter Weise viele einschlägige Materialien übereignet, so von Karl Dietrich Adam/Ludwigsburg; Helmut Bartenstein/Celle; Dieter Berger/Bad Vilbel; Wolf H. Berger/La Jolla; Daniel Bernoulli/Basel; Dieter Betz/Ostfildern; Klaus Blanckenhorn/Köln; Wolf von Engelhardt/ Tübingen; Hans-Joachim Fabian/Fuldabrück; Helmut Fahrion/ Celle; Helmut A. Flügel/Graz; Hugo Genser/Freiburg; Joachim Gersemann/Hannover; Franz Goerlich/Wachtberg; Karl Hiller/ Großburgwedel; Reiner Jordan/Nettlingen; Franz Kockel/Hannover; Martina Kölbl-Ebert/Eichstätt; Rudolf Kohring/Berlin; Peter Kühn/Berlin; Gaston Mayer/ Karlsruhe; Marthe Melguen/Plouzané; Jean Pierre Portmann /Neuchâtel; Werner Prange/Kiel; Ilse Rabien/ Hochheim; Winfried und Brigitte Reiff/Leinfelden; Manfred Schidlowski/Mainz; Siegbert Schueffler und Hermine Schwan/ Erlangen (Nachlass von Werner Schwan); Volker Soergel/Heidelberg (Briefnachlass von Wolfang Soergel); Manfred von Sperber/ Salem; Jörn Thiede/Bremerhaven; Goetz Hellmut Tischer/Bonn; Karl Armin Tröger/ Freiberg; Ida Valeton/Braunschweig; Helmut Weiler/ Mainz; Susanne Weisskopf/Binningen und Kyaw Winn/ Kiel.

\section{Literaturverzeichnis}

Bennett RH, Bryant WR, Hulbert MH (Hrsgb) (1991) Microstructure of fine-grained sediments - From mud to shale. Springer, New York etc: XXII+582 S

Bjerrum L (1954) Geotechnical properties of Norwegian marine clays. Geotechnique 4:46-69

Bjørlykke K (1995) Ivan Th. Koss Rosenqvist. Nachruf im Jahrbuch Norske Videnskaps Akademi; 1-16, mit ausführlichem Literaturverzeichnis

Casagrande A (1932) The structure of clays and its importance in foundation engineering. Boston Soc Civil Engin J 19:168-209

Drost-Hansen W (1991) Some effects of vicinal water on the sedimentation process, compaction and ultimate properties of sediments. In: Bennett et al (1991): 259 - 266

Engelhardt W von, Gaida KH (1963) Concentration change of pore solutions during the compaction of clay minerals. J.Sediment. Petrol. 33:919 - 930

Ferrell RE (1987) The microstructure of clay-rich sediments, Oslofjord. In: Schultz LG, van Olphen Mumpton FA (Hrsgb) (1987) Proc.Intern.Clay Conference Denver 1985:121-127

Ferrell RE Jr., Carpenter PK (1991) Microtexture and microchemistry of clay-rich sediments. In: Bennett et al (1991): 297-301

Føyn E, Rosenqvist IT (1938) Die Suche nach einem unbekannten natürlichen alpha-Strahler. Tidsskr. Kjem.Bergv. 4:64-65

Gipson M (1966) A study of the relations of depth, porosity, and clay mineral orientation in Pennsylvanian shales. J.Sediment. Petrol. 36:888 - 903

Goldschmidt VM (1926) Undersökelser over lersedimenter. Nordisk Jordbrugs Forskning 4-7: 434 - 445

Hulbert MH (1991) Research recommendations of the Clay Microstructure Workshop. In: Bennett et al (1991): 563-566

Kranck K (1991) Interparticle grain size relationships resulting from flocculation. In: Bennett et al (1991)

Mattiat B (1969) Eine Methode zur elektronenmikroskopischen Untersuchung des Mikrogefüges in tonigen Sedimenten. Geol. Jahrbuch Hannover 88:87-111

Mitchell JK (1976) Fundamentals of soil behavior. Wiley New York: $422 \mathrm{~S}$

Moon CF (1972) The microstructure of clay sediments. Earth Sci.Rev. 8:303-321

Norsk Geoteknisk Forening (1974) Retningslinjer for presentasjon av geotekniske undersökelser.Oslo: $16 \mathrm{~S}$

Norsk Krigslexsikon (1940-1945). Darin Artikel „Ivan (Thoralf) Rosenquist", Oslo

O'Brien NR, Slatt RM (1990) Argillaceous rock atlas. Springer New York etc: XV+141 S

Odom IE (1967) Clay fabric and its relation to structural properties in mid-continent Pennsylvanian sediments. J.Sediment.Petrol. $37: 610-623$

Rieke HH III, Chilingarian GV (1974) Compaction of argillaceous sediments. Developments in Sedimentology. Elsevier Amsterdam 16:424 S

Rosenqvist IT (1942) Angående Norske leirers petrografi. Medd.f. Vegdirektoren 3:24-30

Rosenqvist IT (1946 a) Om leirers kvikkaktighet. Medd.f. Vegdirektoren 4:29-36

Rosenqvist IT (1946 b) Om leirers plastisitet. Medd.f. Vegdirektoren $4: 36-41$

Rosenqvist IT (1953) Considerations on the sensitivity of Norwegian Quick clays. Geotechnique 3:195-200

Rosenqvist IT (1955) Investigations in the clay-electrolyte -water system. Nor. Geotekn. Inst. Publ. Oslo 9:125 S

Rosenqvist IT (1956) Die subpolaren Quicktone-Ein Problem der Tonkolloid-Chemie. Geologie Berlin 5/6:495-503

Rosenqvist IT (1958) Remarks to the mechanical properties of soil water systems. Geol.Fören. Förh. Stockholm 80,4:435-457

Rosenqvist IT (1959) Physico-chemical properties of soils - soilwater systems. J.Soil Mech. \& Foundation. Div. Amer. Soc. Civil Eng. (SM 2): $31-53$

Rosenqvist IT (1966) Norwegian research into the properties of Quick clay-a review. Eng.Geol. 1, 16: 445-450 
Rosenqvist IT (1977) A general theory of Quick clay properties. Proc.Third European Clay Conference, Oslo: 215-228

Smalley LL (1970) Nature of Quick clay. Nature 231:310

Spears DA (1976) The fissility of some Carboniferous shales. Sedimenology 23:721-725

Syvitski IPM (1991) The changing microfabric of suspended particulate matter-The fluvial to marine transition: Flocculation, agglomeration, and pelletization. In: Bennett et al (1991): 131137

Terzaghi K (1925) Erdbaumechanik auf bodenphysikalischer Grundlage. Leipzig/Wien: 399 S

Torrance JK (1983) Towards a general model of Quick clay development. Sedimentology 30:547-555 